This is the author's final, peer-reviewed manuscript as accepted for publication. The publisher-formatted version may be available through the publisher's web site or your institution's library.

\title{
Psychosocial and demographic correlates of objectively measured physical activity in structured and unstructured after-school recreation sessions
}

Richard R. Rosenkranz, Greg J. Welk, Tanis J. Hastmann, and David A. Dzewaltowski

\section{How to cite this manuscript}

If you make reference to this version of the manuscript, use the following information:

Rosenkranz , R. R., Welk, G.J., Hastman, T.J., \& Dzewaltowski, D.A. (2011).

Psychosocial and demographic correlates of objectively measured physical activity in structured and unstructured after-school recreation sessions. Retrieved from http://krex.ksu.edu

\section{Published Version Information}

Citation: Rosenkranz , R. R., Welk, G.J., Hastman, T.J., \& Dzewaltowski, D.A. (2011). Psychosocial and demographic correlates of objectively measured physical activity in structured and unstructured after-school recreation sessions. Journal of Science and Medicine in Sport, 14(4), 306-311.

Copyright: Copyright @ 2011 Sports Medicine Australia Published by Elsevier Ltd

Digital Object Identifier (DOI): doi:10.1016/j.jsams.2011.01.005

Publisher's Link: http://www.sciencedirect.com/science/article/pii/S1440244011000259

This item was retrieved from the K-State Research Exchange (K-REx), the institutional repository of Kansas State University. K-REx is available at http://krex.ksu.edu 
Title: Psychosocial and demographic correlates of objectively measured physical activity in structured and unstructured after-school recreation sessions

Richard R. Rosenkranz, ${ }^{1,2}$ Greg J Welk, ${ }^{3}$ Tanis J Hastmann, ${ }^{2,4}$ and David A Dzewaltowski ${ }^{4}$ ${ }^{1}$ School of Biomedical and Health Sciences, University of Western Sydney, Sydney, Australia

${ }^{2}$ Department of Human Nutrition, Kansas State University, Manhattan, KS, USA ${ }^{3}$ Department of Kinesiology \& Nutrition and Wellness Research Center, lowa State University, Ames, IA, USA

${ }^{4}$ Department of Kinesiology, Kansas State University, Manhattan, KS, USA

Running head: After-school PA correlates

\footnotetext{
Suggested citation: Rosenkranz, R.R., Welk, G.J., Hastmann, T.J., \& Dzewaltowski, D.A. (2011). Psychosocial and demographic correlates of objectively measured physical activity in structured and unstructured after-school recreation sessions. Journal of Science and Medicine in Sport, 14:306-311. doi: 10.1016/j.jsams.2011.01.005
} 


\section{$\underline{\text { Abstract }}$}

Most studies of psychosocial and demographic correlates of physical activity (PA) have examined relationships across various types of physical and social environments, rather than within a specific environmental behavior setting.

Objectives: The objective of this study was to investigate correlates of PA in structured and unstructured after-school recreation sessions.

Design: This study is cross-sectional.

Methods: School records, questionnaires, and anthropometry were used to obtain demographic and psychosocial variables. Third and fourth-grade children $(n=230)$ from seven schools wore Actigraph GT1M accelerometers up to six times per year during after-school programming. Accelerometer data were processed to determine percentage of time in moderate-to-vigorous PA (T scores, reflective of an individual child's PA level relative to group mean, were computed for each session and averaged across sessions). Pearson correlations, point-biserial correlations, and mixed-model analyses were used to determine significant associations with PA for each session type (structured and unstructured).

Results: For structured sessions, gender, PA barriers self-efficacy, and PA enjoyment were significantly related to PA. For unstructured sessions, only gender was related to PA. Conclusions: Despite equivalent opportunities to participate in active recreation, boys were more active than girls, and children varied in PA level partly due to psychosocial factors. Our results showed that PA self-efficacy and enjoyment explained variability in structured PA sessions.

Key words: children, physical activity, exercise, accelerometer, after school, determinants 


\section{$\underline{\text { Introduction }}$}

Physical activity is associated with numerous positive health outcomes in children and adolescents. ${ }^{1}$ Despite the myriad benefits of PA, many children do not accumulate amounts of moderate to vigorous PA sufficient to meet current guidelines. ${ }^{2}$ Furthermore, PA tends to decline from childhood to adolescence, so youth may decrease over time from already insufficient amounts. ${ }^{2,3,4}$

Understanding the correlates and determinants of PA is important for public health efforts seeking to increase or maintain PA behaviors of children and adolescents. Some nonmodifiable correlates (e.g., age) are useful to identify intervention target populations. Modifiable correlates (e.g., enjoyment) can be useful for intervention development. Currently, much is known regarding correlates of child and adolescent PA, and several excellent reviews are available in the literature. ${ }^{3,4,5}$ Self-efficacy, one's confidence to perform a specific behavior (e.g., being physically active), and enjoyment, the degree to which one finds PA to be pleasant, are consistently identified PA correlates in youth. ${ }^{3}$ Opportunities to be active, parental (social) support for PA and direct parental help for PA have also been previously identified as related to youth PA. ${ }^{3}$

Most correlate studies, however, have focused on multi-day or habitual (free living) PA. ${ }^{6,7}$ Habitual PA is important, but correlate studies investigating links between psychosocial variables and habitual PA may be biased by confounds such as socioeconomic status that could influence the type and amount of environmental opportunities for physical activity. An ecological framework ${ }^{8}$ looks not only at intrapersonal (biological and psychological) factors, but also interpersonal, cultural, organizational, physical environmental, and policy factors to explain or predict behavior. Such a framework suggests that a child's PA is a function of multi-level environmental opportunities for participation and individual-level determinants of taking advantage of such opportunities. Compared to the robust literature using habitual PA measurement, fewer studies have assessed psychosocial and demographic correlates of PA 
when children have apparently equal micro-level environmental participation opportunities. ${ }^{9}$ Discrete PA behavior settings, such as school recess and recreation sessions held at afterschool programs, provide examples of micro-level environments and could be examined to reveal less biased relationships between psychosocial and demographic variables and PA participation. Previous research has shown that some after-school programs contribute around one third of the moderate-to-vigorous PA needed to meet daily recommendations in attending kids, and after-school time is generally seen as a time conducive to PA participation. ${ }^{10}$ Identifying PA influences within such settings may help identify psychosocial attributes to be targeted through interventions promoting 60 minutes of daily PA. The purpose of this study was to investigate the psychosocial and demographic correlates of PA in structured and unstructured after-school recreation sessions (e.g., periods when children played outside, did sports or physically active games). We hypothesized that those children with higher levels of PA task and barriers self-efficacy, PA enjoyment, perceived PA opportunity, and perceptions of habitual PA would be more physically active in after school recreation sessions. We hypothesized that afterschool PA would be influenced by gender and weight status, and further hypothesized that correlates of PA would differ by session type (i.e., structured or unstructured).

\section{Methods}

This study includes secondary data analyses from third- and fourth-grade children $(\mathrm{N}=$ 230) attending one of seven after-school programs in the Healthy Opportunities for Physical Activity and Nutrition (HOP'N) After-School Project. HOP'N was a three-year randomized controlled trial, with a primary purpose to prevent obesity through staff training, increased opportunities for PA and healthful eating, and an educational curriculum delivered in the afterschool setting. The present study is cross-sectional, assessing associations between psychosocial/demographic variables and PA in 140 active recreation sessions of the fourthgrade group, within each observation of after-school programs (three control sites and four 
intervention sites) over three years. Children's parents signed a consent form prior to data collection, and children assented to participate. This study was approved by the USD 497 research committee and the IRB at Kansas State University.

Height and weight measurements were obtained from children by trained research assistants, and used to compute body mass index (BMI) percentiles as described previously. ${ }^{10}$ Children with a BMI exceeding the $85^{\text {th }}$ percentile were classified as overweight. PA was objectively measured using Actigraph GT1M uniaxial accelerometer-based monitors (Shalimar, FL), storing count data in 30-s epochs, as previously reported. ${ }^{10}$ Briefly, research assistants travelled to each site to collect PA data during six days per academic year, over three years (2005-2008). Participating children wore Actigraph monitors for the whole after-school day.

During after school program time (typically $2 \mathrm{hr}$ ), research assistants recorded fourthgrade group activities as sessions (activity the majority of children were doing) in a customized log book, noting each session's start/stop times. Sessions were coded into these categories: 1) Active recreation; 2) Enrichment; 3) Academic time; 4) Non-active recreation; and 5) Snack. Active recreation sessions averaged 25 minutes in duration, with 18 children per session. Active recreation predominantly took place in gymnasiums or outside playgrounds, and were coded as structured $(\mathrm{N}=72)$ for games or group activities (e.g., basketball), or as unstructured $(N=68)$. Unstructured sessions allowed children access to sport and playground equipment for use at children's discretion. After school programs always offered at least one active recreation session per day, and research assistants coded separate sessions for cases where structured and unstructured sessions were contiguous to each other. Upon program day ending, all Actigraphs and logs were returned to the laboratory for downloading and storage.

Accelerometer count data for each child in each session were processed to determine percentage of session time spent in moderate-to-vigorous PA ( $\geq 4$ metabolic equivalents). The age-specific counts per minute threshold (divided by two for 30-s epoch) for four METs was derived from Freedson and colleagues' equation. ${ }^{11}$ Session codes (as listed above), session 
start/stop times, child attendance, and start/stop of accelerometer wear times were used to ensure only data of attending children wearing monitors were analyzed. Because we examined variability in PA under controlled conditions, data were included only if youth were present for the whole session. The percentage of session time spent in moderate-to-vigorous PA was computed for each session to control for variability in session length. These percentages were then converted to T-scores to reflect the child's activity level in a session, relative to the group mean. ${ }^{9}$ The group mean T-score is 50 , with a standard deviation of 10 for each session at each school, and individual children then get a T-score that shows how they compare to the group mean for each session. This use of T-scores controls for variability by session and site in the type and intensity of activity performed, allowing relative comparisons among children. Each child's collection of T-scores was averaged across sessions of participation, providing one PA variable per child. At least two sessions of active recreation were required for a child's data to be included in the final analyses.

Gender, ethnicity, and socioeconomic status were obtained from school records. Psychosocial variables were measured via questionnaire during after-school program time at the academic year's beginning and end. Both scores for each individual were averaged to provide a single score for analysis. Parents were sent questionnaires to complete at home, and children completed questionnaires as a group at the after-school site. Following a script, instructions and questions were read aloud to all children, with a poster board displaying written definitions and example questions. PA task self-efficacy was assessed with one item: "How sure are you that you can do physical activity 60 minutes each day?" Children responded on a three-point scale: Not sure at all - Somewhat sure - Very Sure. PA barriers self-efficacy was assessed with four items (Cronbach's $\alpha=.64$ ): e.g., "How sure are you that you can be physically active, no matter how tired you may feel?" Children responded on a three-point scale as above. PA enjoyment was assessed with two items (Cronbach's $\alpha=.72)$ : "I enjoy the different kinds of physical activities or sports offered at the after-school program" and "I think the 
types of physical activities or sports offered at the after-school program are fun." Children responded on a three-point scale: Yes - Don't know - No. Perceived opportunity for PA was assessed with two items (Cronbach's $\alpha=.49$ ): "There are a lot of chances for kids to get involved in physical activity at the after-school program" and "We are satisfied with the physical activities or sports offered at the after-school program." Children responded on a similar threepoint scale. Perceived habitual PA was assessed using a two-item screener. ${ }^{12}$ Scores were averaged and dichotomized into meeting PA guidelines (60 minutes PA $\geq 5$ days/week) or not. Parental social support for physical activity was assessed with five items ${ }^{13}$ (Cronbach's $\alpha=.83$ ): Parents responded on a six-point scale from never to daily. To accommodate different scales among variables and potential problems from multi-collinearity, psychosocial variables were standardized by taking each variable score minus the mean and dividing by the standard deviation.

Descriptive statistical analyses were performed using SPSS (version 15.0; Chicago, IL). Inferential statistical analyses were performed using SAS (version 9.2; Cary, NC). Analogous to the methods of Trost and colleagues, ${ }^{14,15}$ separate session-specific Pearson correlations (and point-biserial correlations) were used to assess the unadjusted associations between demographic and psychosocial variables and mean PA T-score. Session-specific mixed-model analyses (Proc Mixed) were run (complete case analysis) to assess relationships of demographic and psychosocial variables with PA T-score, adjusting for the potential clustering effect of schools. In the mixed model, school site was a random effect and child gender, ethnicity, socioeconomic status, and overweight status were covariates. Main effects analyzed included intervention condition, study year, and those psychosocial variables with significant Pearson correlation coefficients in the unadjusted analyses. For all analyses, alpha was set at 0.05. In cases where a child participated across multiple years, only that child's first-year data were used. 


\section{$\underline{\text { Results }}$}

Our sample $($ mean age $=9.5$ years, SD $=0.7)$ included boys $(49 \%)$, girls $(51 \%)$ third graders $(22 \%)$, fourth graders $(78 \%)$, Caucasians $(60 \%)$ minorities $(40 \%)$, and diverse socioeconomic status (54\% eligible for free/reduced lunch). Approximately $36 \%$ of the sample had an overweight or obese BMI value.

Structured recreation sessions: Among demographic variables, point-biserial and Pearson correlations revealed a medium-sized association between PA T-score and gender $\left(r_{p b}\right.$ $=0.39, p<0.001)$, but no significant associations with BMI, ethnicity, or socioeconomic status ( $p$ $>0.05$ ). Pearson correlations assessing the unadjusted relationships between PA T-score and psychosocial variables in structured recreation sessions are shown in Table 1. Analyses revealed small to medium-sized correlations for PA task self-efficacy $(r=0.18, p=0.014) \mathrm{PA}$ barriers self-efficacy $(r=0.30, p<0.001)$ and PA enjoyment $(r=0.21, p=0.005)$, and nonsignificant associations for PA opportunities, perceived habitual PA, and PA parental social support $(p>0.05)$.

Next, mixed-model analyses were run, using variables having significant unadjusted associations with PA T-score (See table 2). Type 3 tests of fixed effects showed gender $(F=$ 23.8, $p<.001)$, PA barriers self-efficacy $(F=6.1, p=0.015)$, and PA enjoyment $(F=5.0, p=$ 0.027), made significant contributions to the model. In these analyses, the following variables were not significant: Ethnicity $(F=2.6, p=0.111)$; socioeconomic status $(F=0.1, p=0.812)$; weight status $(F=0.4, p=0.554)$; PA task self-efficacy $(F=0.4, p=0.509)$; $y$ ear $(F=0.7, p=$ $.423)$; and condition $(F=1.7, p=.197)$.

Unstructured recreation sessions: Among demographic variables, point-biserial and Pearson correlation analyses revealed a medium-sized association between PA T-score and gender $\left(r_{p b}=0.43, p<0.001\right)$, but not BMI, ethnicity, or socioeconomic status $(p>0.05)$. Pearson correlations assessing unadjusted relationships between PA T-score and psychosocial variables in structured recreation sessions are shown in Table 1. These revealed small 
correlations for PA barriers self-efficacy $(r=0.19, p=0.013)$, PA parental social support $(r=$ $0.21, p=0.018)$, and non-significant correlations for PA task self-efficacy, PA enjoyment, PA opportunities, and perceived habitual PA $(p>0.05)$.

Next, mixed-model analyses were run using variables having significant unadjusted correlations with PA T-score (See table 2). Type 3 tests of fixed effects showed that gender ( $F$ $=26.2, p<0.001)$ made a significant contribution to the model. In the same analyses, the following variables were not significant: Socioeconomic status $(F=0.7, p=0.400)$; ethnicity $(F=$ $0.5, \mathrm{p}=0.504)$; weight status $(F=0.2, p=0.685)$; PA task self-efficacy $(F=0.6, p=0.434) ; \mathrm{PA}$ barriers self-efficacy $(F=2.0, p=0.160)$; year $(F=0.8, p=0.387)$; and condition $(F=0.5, p=$ $0.462)$.

\section{Discussion}

This study investigated the psychosocial and demographic correlates of PA, separately for structured and unstructured after-school program recreation sessions over three years. Our main finding was that when provided equivalent opportunities to do moderate-to-vigorous PA in a variety of recreation sessions, PA level consistently varied by gender, and by key psychosocial variables in structured sessions. Despite similar participation opportunities, boys were more active than girls in both session types. Our results showed that PA barriers selfefficacy and PA enjoyment both explained unique variability during structured PA sessions. These results provide less biased associations than correlate studies conducted under free living conditions, because all children had apparently equal opportunities to be active. The findings support the importance of self-efficacy (particularly PA barriers self-efficacy) for explaining PA behavior because youth with higher scores exhibited higher levels of PA relative to their peers. The results also support the importance of enjoyment, because this predicted the amount of activity that children obtained during the structured sessions. 
Gender differences in PA of children and adolescents are among the most robust of findings in the literature on correlates of PA. Studies have generally assessed habitual (free living) physical activity, but nearly all studies show girls to be less active than boys. ${ }^{3,4}$ Our group's previous study of after-school program PA ${ }^{10}$ showed that boys accumulated about six minutes more of moderate-to-vigorous PA over a two-hour period, and the present study confirmed that relationship for the subset of recreation time, and over additional years of study. The novelty of the present finding is that the relationship was consistent between structured and unstructured sessions, even when boys and girls had equivalent opportunities for PA. This consistent difference between boys and girls in PA suggests that public health interventions may need to consider providing additional PA opportunities or time in active recreation programs for girls, beyond that provided to boys, in order to achieve adequate daily PA.

PA enjoyment has been previously identified as a correlate of PA in some studies, particularly for girls, ${ }^{5}$ but two literature reviews have concluded that there is no consistent relationship across studies for children and adolescents. ${ }^{3,4}$ Our study found enjoyment to be positively associated with PA in structured sessions, but not for unstructured sessions. The distinction is important, because it may help explain the discrepant findings in the literature on PA correlates. A previous study ${ }^{9}$ using a similar design in a summer camp also found enjoyment ("Attraction to Physical Activity") to be the most salient predictor of children's activity. This previous study emphasized structured activities, but clear distinctions were not made between structured versus unstructured opportunities. ${ }^{9}$ Based on our findings, enjoyment of specific contexts and activities may be important considerations in the relationship with PA, rather than more global PA enjoyment. Further work with more refined measures of context-specific PA enjoyment will be needed to assess the degree to which enjoyment is related to PA. Our results suggest, however, that leaders of after-school programs should be encouraged to select enjoyable activities for structured recreation sessions, or to take steps to make selected activities more enjoyable for attending children. 
Self-efficacy is a key construct in Social Cognitive Theory, ${ }^{16}$ and is frequently identified as a correlate and potential mediator of youth habitual PA., ${ }^{4,5}$ the present study, both PA task and barriers self-efficacy variables appeared influential in unadjusted analyses for the two session types. However, the mixed-model analyses revealed that PA barriers self-efficacy was only related to PA in structured sessions. Specifically, children who had higher levels of confidence in their abilities to be physically active despite barriers were more active in structured sessions, compared to their less-confident peers. This confidence to be physically active despite barriers could be partly responsible for higher levels of PA in recreation sessions. However, it is also possible that generally active children chose to be more active in these sessions, and reported higher PA barriers self-efficacy. Generally inactive children may report lower PA barriers self-efficacy, and may be less active in structured sessions. It was unexpected that PA barriers self-efficacy contributed to models for structured PA but not unstructured PA. It would seem more logical for barriers to exist in unstructured settings, but the provision of equipment and space may have made it easy for youth to choose to be active. PA Task selfefficacy was not found to contribute to either model, but this may be due to the way it was assessed relevant to habitual PA (confidence to do PA 60 minutes each day). Regardless, there appear to be distinctions in relationship between PA and self-efficacy that vary by type of self-efficacy and type of physically active setting, and these warrant further investigation. Interventions set in after-school structured PA programs should consider including components designed to foster barriers self-efficacy as a potential mediator of PA. The present results support interventions aimed at promoting self-efficacy, particularly barriers self-efficacy, to influence physical activity behavior in youth who participate in after school program recreation sessions.

Limitations of the present study include its cross-sectional design, as this precludes evaluation of temporality or causality in the associations. Future work on this topic with longitudinal designs could provide insight into the influence of correlates over time. Also, some 
of our psychosocial measures were based on one or two questionnaire items, and may be limited in psychometric properties, including reliability and validity. Better measures could improve the ability to determine relationships between variables. Lastly, our primary outcome variable, PA T-score, provides a measure of child PA that is relative to the group in a session, not an absolute measure of PA. This makes it robust for comparisons within and between cohorts, but less interpretable with regard to meeting daily PA standards. Counterbalancing these limitations were notable strengths. We used rigorous methods to obtain objective measures of PA and demographic variables. We obtained data over fall, winter, and spring seasons in 140 recreation sessions, including a wide variety of activities, locations, and children, over a three-year period of study.

\section{Conclusions}

In after school recreation sessions with apparently equal opportunities to participate in PA, boys and children with higher levels of self-efficacy (particularly efficacy to overcome PA barriers) and enjoyment were more physically active. Findings did not support hypothesized influences for weight status or perceived PA opportunities, social support, and perceived habitual PA. A novel finding is that psychosocial and demographic correlates appear to differ as a function of after-school active recreation session structure. This suggests that different intervention strategies and approaches may be needed for promoting activity in structured versus unstructured sessions.

\section{Practical implications}

- Developers of children's physical activity interventions should consider enjoyment and PA barriers self-efficacy as potential mediators of physical activity behavior in structured recreation sessions. 
- To derive a level of PA similar to that of boys in after-school program recreation sessions, girls may need additional or extended PA opportunities.

\section{$\underline{\text { Acknowledgements }}$}

This study was supported by NRI Grant \#2005-35215-15418 from the USDA

Cooperative State Research, Education, and Extension Service Human Nutrition and Obesity Program. We thank Stewart Trost, Karla Foster and Sara Rosenkranz for assisting with data collection and management. We thank the people of USD 497 and Boys and Girls Club in Lawrence, KS for their participation and assistance. 
Table 1. Pearson Correlation Matrix for Study Variables in Structured $(N=181)$ and Unstructured ( $N=173)$ After-School PA Recreation Sessions

\begin{tabular}{|c|c|c|c|c|c|c|c|}
\hline Variables- Structured & 1 & 2 & 3 & 4 & 5 & 6 & 7 \\
\hline 1. Session PA T-score & 1.00 & & & & & & \\
\hline 2. PA task self-efficacy & $.18^{*}$ & 1.00 & & & & & \\
\hline 3. PA barriers self-efficacy & $.30^{*}$ & $.76^{*}$ & 1.00 & & & & \\
\hline 4. PA Enjoyment & $.21^{*}$ & $.17^{*}$ & $.18^{*}$ & 1.00 & & & \\
\hline 5. Perceived PA opportunities & .06 & .05 & .05 & $.34^{*}$ & 1.00 & & \\
\hline 6. Perceived habitual PA & .07 & $.18^{*}$ & $.25^{*}$ & .03 & .08 & 1.00 & \\
\hline 7. Parent PA social support & .17 & $.26^{*}$ & $.31^{*}$ & .16 & .01 & .13 & 1.00 \\
\hline Variables- Unstructured & 1 & 2 & 3 & 4 & 5 & 6 & 7 \\
\hline 1. Session PA T-score & 1.00 & & & & & & \\
\hline 2. PA task self-efficacy & .11 & 1.00 & & & & & \\
\hline 3. PA barriers self-efficacy & $.19^{*}$ & $.80^{*}$ & 1.00 & & & & \\
\hline 4. PA Enjoyment & .05 & $.26^{*}$ & $.22^{*}$ & 1.00 & & & \\
\hline 5. Perceived PA opportunities & .03 & $.17^{*}$ & $.20^{*}$ & $.39^{*}$ & 1.00 & & \\
\hline 6. Perceived habitual PA & .01 & .13 & $.21^{*}$ & .06 & .05 & 1.00 & \\
\hline 7. Parent PA social support & $.21^{*}$ & $.20^{*}$ & $.27^{*}$ & .12 & .02 & .12 & 1.00 \\
\hline
\end{tabular}

${ }^{*}$ significant correlation, $p<.05$ 
Table 2: Results of mixed model analyses examining factors contributing to the PA T-score

\begin{tabular}{l|l|l}
\hline Model components & Structured Sessions & Unstructured Sessions \\
\hline Gender & $3.1^{*}$ & $5.5^{*}$ \\
\hline Ethnicity & 1.1 & -0.8 \\
\hline Socioeconomic Status & 0.2 & -1.0 \\
\hline Overweight Status & 0.4 & -0.4 \\
\hline PA Task Self-Efficacy & & -0.7 \\
\hline PA Barriers Self-Efficacy & -0.3 & 1.2 \\
\hline PA Enjoyment & $1.2^{*}$ & --- \\
\hline Parent PA Social Support & $0.7^{*}$ & 0.6 \\
\hline Year of Study & -- & -0.6 \\
\hline Intervention Condition & & 0.8 \\
\hline & 0.3 & $49.3^{*}$ \\
\hline Intercept & 1.1 & \\
\hline
\end{tabular}

Unstandardized coefficients reflecting the amount of change in PA T-score for each 1 point change in the variable. ${ }^{*} p<0.05$ 


\section{References}

1. Strong WB, Malina RM, Blimkie CJ, et al. Evidence based physical activity for school-age youth. J Pediatr. 2005; 146(6):732-7.

2. Troiano RP, Berrigan D, Dodd KW, et al. Physical activity in the United States measured by accelerometer. Med Sci Sports Exerc. 2008; 40(1):181-8.

3.Sallis JF, Prochaska JJ, Taylor WC. A review of correlates of physical activity of children and adolescents. Med Sci Sports Exerc. 2000; 32(5):963-75.

4. Van der Horst K, Paw MJ, Twisk JWR, et al. A brief review on correlates of physical activity and sedentariness in youth. Med Sci Sports Exerc. 2007; 39(8):1241-50.

5. Biddle SJH, Whitehead SH, O'Donovan TM, et al. Correlates of Participation in Physical Activity for Adolescent Girls: A Systematic Review of Recent Literature. J Phys Act \& Health. 2005; 2(4):423-434.

6. Cardon G, Philippaerts R, Lefevre J, et al. Physical activity levels in 10- to 11-year-olds: clustering of psychosocial correlates. Public Health Nutr. 2005; 8(7):896-903.

7. Strauss RS, Rodzilsky D, Burack G, et al. Psychosocial correlates of physical activity in healthy children. Arch Pediatr Adolesc Med. 2001; 155(8):897-902.

8. Sallis JF, Cervero RB, Ascher W, et al. An ecological approach to creating active living communities. Annu Rev Public Health. 2006; 27:297-322.

9. Welk GJ, Schaben JA. Psychosocial correlates of physical activity in children-a study of relationships when children have similar opportunities to be active. Meas Phys Educ Exerc Sci. 2004; 8(2):63-81. 
10. Trost SG, Rosenkranz RR, Dzewaltowski D. Physical Activity Levels among Children Attending After-School Programs. Med Sci Sports Exerc. 2008; 40(4):622-9.

11. Freedson P, Pober D, Janz KF. Calibration of accelerometer output for children. Med Sci Sports Exerc. 2005; 37(11 Suppl):S523-30.

12. Prochaska JJ, Sallis JF, Long B. A physical activity screening measure for use with adolescents in primary care. Arch Pediatr Adolesc Med. 2001; 155(5):554-9.

13. Trost SG, Sallis JF, Pate RR, et al. Evaluating a model of parental influence on youth physical activity. Am J Prev Med. 2003; 25(4):277-82.

14. Trost SG, Pate RR, Dowda M, et al. Psychosocial correlates of physical activity in white and African-American girls. J Adolesc Health. 2002; 31(3):226-33.

15. Trost SG, Pate RR, Ward DS, et al. Correlates of objectively measured physical activity in preadolescent youth. Am J Prev Med. 1999; 17(2):120-6.

16. Bandura A. Health Promotion by Social Cognitive Means. Health Educ \& Behav. 2004; 31(2):143-64. 